\title{
La pollution de l'eau par les produits phytosanitaires : état et origine
}

\author{
M Schiavon ${ }^{1,2^{\star}}$, C Perrin-Ganier ${ }^{1}$, JM Portal 2 \\ ${ }^{1}$ École nationale supérieure d'agronomie et des industries alimentaires, BP 172, F54505 Vandœuvre-lès-Nancy; \\ 2 CNRS, centre de pédologie biologique, BP 5, F54501 Vandœuvre-lès-Nancy, France
}

(Reçu le 25 août 1994 ; accepté le 16 juin 1995)

\begin{abstract}
Résumé - Les campagnes d'analyse des eaux de surface ou souterraines utilisées comme ressource en eau potable montrent fréquemment une pollution par les produits phytosanitaires au-delà de la norme UE. Cette situation est en partie due à une agriculture intensive, au non respect des bonnes pratiques agricoles, aux usages non agricoles des pesticides mais également à l'existence de sites particulièrement vulnérables (réseau hydrologique dense et diffus, relief accidenté, sols filtrants ou fissurés, nappes peu profondes). Son évaluation peut être réalisée à différentes échelles. Cependant, seule l'étude régionale apparaît comme satisfaisante. En effet, elle permet de mieux cibler les produits à rechercher, les dates d'échantillonnage et le type d'eau à surveiller en fonction des ressources en eau potable. Les pesticides mis en cause dans la pollution de l'eau en France sont actuellement peu nombreux : atrazine, simazine, lindane, carbofuran, phénylurées. Mais cette liste peut se diversifier en prenant en compte la spécificité de l'agriculture locale par réduction de l'échelle d'investigation ou par la prise en compte des produits de dégradation des pesticides utilisés (dééthylatrazine). Pour les eaux de surface, la pollution peut être très élevée mais de courte durée. Elle est étroitement liée aux possibilités de transport des pesticides par ruissellement et à l'effet de dilution par les eaux provenant de secteurs non traités. En revanche, pour les eaux souterraines, la pollution est plus discrète mais elle peut être permanente. Elle est régulée par le processus de lessivage, lui-même lié à la disponibilité du produit et à la dynamique de l'eau. Dans le cas où le pesticide est à la fois persistant et quelque peu mobile, il peut y avoir un stockage du produit dans le sol, qui entretient la pollution des eaux souterraines.
\end{abstract}

\section{pollution / pesticide / eau / sol}

Summary - The pollution of water by pesticides: state and origin. In Europe, monitoring campaigns of surface and groundwaters used for drinking water frequently show pollution by pesticides, above the EU specification. This situation is due to intensive agriculture, non-respect of good agricultural practices, non-agricultural uses and the existence of especially vulnerable sites (eg, thick and diffuse hydrological system, broken relief, filtering or cracked soils, water table lying near the surface). The assessment of the pollution can be conducted at different levels. However, local study appears to be the only satisfactory method. Indeed, it allowed us to focus on some pesticides, sampling dates and water supplies, in relation to the local characteristics. In France, few pesticides are involved in water pollution: atrazine, simazine, lindane, carbofuran and phenylureas. However, this list would be different if practices carried out locally or degradation products (deethylatrazine) were taken into account. The pollution of surface water can reach a high level but for a small time. It is closely related to the ability of pesticides to be transported by runoff, and the dilution with water from untreated areas. In contrast, groundwater is weakly but, in some instances, continuously polluted.

\footnotetext{
${ }^{\star}$ Correspondance et tirés à part
} 
This depends on the leaching processes, and, hence, on the availability of pesticides and the water dynamics. In the case of pesticides with high persistance and relatively good mobility, the storage of pesticides in soil sustains the groundwater pollution.

pollution / pesticide / water / soil

\section{INTRODUCTION}

L'attention portée à l'état de pollution de l'eau par les produits phytosanitaires en France est relativement récente. Aussi, toutes les actions d'envergure menées au niveau national sont postérieures à la directive CEE $n^{\circ} 80 / 778$ et au décret $N^{\circ} 83-3$ au 3 janvier 1989.

Cette pollution prend son origine dans l'utilisation annuelle de près de $80000 t$ de produits phytosanitaires (Coste, 1990). Même si la SNCF, les Ponts et Chaussées, l'industrie ou les particuliers amateurs de gazons sont d'importants utilisateurs (Montiel, 1990), la majeure partie de ces substances est employée par l'agriculture intensive. Près de 500 matières actives sont commercialisées, mais la fréquence et les quantités utilisées sont très inégales. En fait, la situation est aggravée par la place prépondérante de quelques cultures dans chaque région. Cela entraîne l'emploi presque exclusif de certaines molécules particulièrement performantes. C'est le cas de l'atrazine, étroitement associée à la culture du maïs ou de l'isoproturon sur céréales d'hiver infestées de vulpin.

Quel que soit le type de traitement (pré-semis, post-semis, foliaire...), c'est le sol qui, in fine, reçoit directement ou indirectement la presque totalité des produits utilisés. Aussi, en dehors des pollutions directes (vidange de fonds de cuves dans les cours d'eau ou les puits désaffectés, dérives d'embruns...), la pollution de l'eau est liée aux interactions pesticide-sol et modulée par les conditions climatiques. Le caractère polluant d'un produit est, en pratique, associé à l'incapacité du sol à le retenir et à le dégrader avant que, sous l'effet de l'eau, il ne soit dispersé dans l'environnement. En schématisant l'analyse, on peut estimer que les possibilités de pollution de l'eau par un produit phytosanitaire sont corrélées à son état de disponibilité dans le sol au cours du temps (Barriuso et al, 1994) et à la dynamique de l'eau.

Nous nous proposons ici, non pas de recenser l'ensemble des résultats des travaux entrepris en vue de dresser un état des lieux de la pollution de l'eau par les produits phytosanitaires, mais simplement d'examiner quelques cas afin de situer le degré de contamination en France par rapport à d'autres pays, d'évoquer l'intérêt de dresser de tels constats et de soulever les aspects de la recherche qui devront être abordés pour préjuger de l'évolution du niveau de pollution des eaux.

\section{ÉCHELLE DE L'ÉTAT DES LIEUX}

L'évaluation de la pollution de l'eau par les produits phytosanitaires représente, du point de vue toxicologique du moins, un enjeu considérable. De ce fait, il apparaît comme indispensable d'avoir une image de la situation française. Bien que cela représente un travail important, une étude d'ampleur nationale a été réalisée par la Lyonnaise des Eaux à l'initiative de l'Union des industries de la protection des plantes (UIPP). Sur 1929 résultats issus de 11 sites de prélèvements d'eaux souterraines et 2 sites de prélèvement d'eaux de surface répartis sur l'ensemble de la France et échantillonnés à différentes périodes de l'année, $99,73 \%$ des échantillons présentaient une teneur en résidus inférieure à la limite de potabilité : $0,1 \mu \mathrm{g} . \mathrm{I}^{-1}$. Parmi ces échantillons, dans $99,06 \%$ des cas, les 38 matières actives recherchées n'étaient pas décelables (My, 1990). Ces chiffres donnent une première impression rassurante, à la fois pour les eaux souterraines et de surface. En fait, l'examen détaillé des résultats publiés par Legrand et al (1991), qui ont vraisemblablement donné lieu à cette analyse (tableau I), montre une pollution non négligeable de 9 captages sur 11 et une contamination sévère des eaux des rivières Seine et Marne.

L'analyse des résultats peut donc être très subjective. En outre, l'introduction dans la liste des produits recherchés d'un grand nombre de molécules très peu utilisées conduit, de manière évidente, à de faux résultats rassurants.

En définitive, une meilleure appréciation sera obtenue à partir des études à caractère régional où la liste des produits à rechercher sera établie en fonction des pratiques culturales locales et sera ciblée sur ceux particulièrement utilisés. Cette échelle d'investigation permet par ailleurs un meilleur choix des périodes de prélèvement 
Tableau I. Matières actives dosées au cours de 5 campagnes d'analyse pour 13 sites couvrant les zones agricoles les plus significatives de France (d'après Legrand et al, 1991).

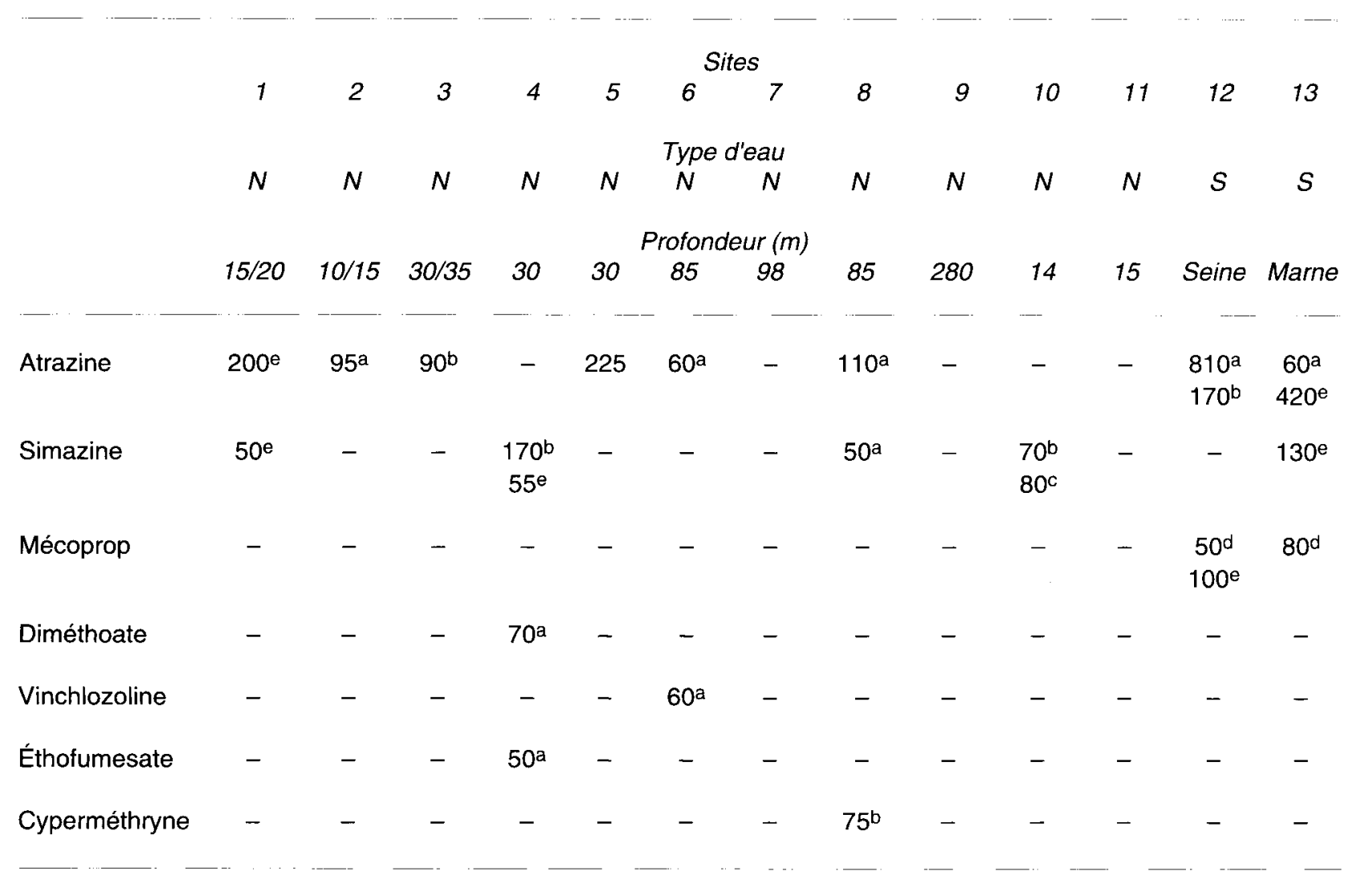

Valeurs exprimées en ng. $I^{-1} . \mathrm{N}$ : nappe; $\mathrm{S}:$ surface; a $: 1^{\text {re }}$ campagne (juillet 1987) ; ${ }^{b}: 2^{e}$ campagne (nov 1987 ) c $^{c}: 3^{e}$ campagne (mars 88 ) ; ${ }^{d}: 4^{\text {e }}$ campagne (juin 1988); e : $5^{e}$ campagne (avril 1989).

qui sont d'une importance capitale, en particulier pour les eaux de surface où le transfert du produit de la parcelle au cours d'eau est direct lors des épisodes de ruissellement.

\section{NIVEAUX DE CONTAMINATION DES EAUX DE SURFACE}

Différents mécanismes sont à l'origine de la pollution des ressources en eau potable. II s'agit principalement du ruissellement dans le cas des captages de surface et du lessivage dans le cas des captages souterrains. Ces 2 situations seront présentées successivement ici, à partir d'exemples à échelle régionale qui sont le mieux appropriés.

\section{Cas de la Bretagne}

Nous reprenons ici les résultats publiés par Gillet (1992). Les ressources en eau potable de cette région sont essentiellement superficielles. Ceci explique, en partie, la mise en place d'un plan régional de surveillance de 5 rivières (Aven, Arguenon, Oust, Seiche et Vilaine) collectant les eaux de 5 bassins versants occupés par une agriculture intensive $(25 \%$ de maïs, $25 \%$ de céréales) utilisatrice entre autres de $630 \mathrm{t}$ d'atrazine et de $5 \mathrm{t}$ de simazine. Dans le cadre de la pollution de l'eau, il faut également prendre en compte $6 \mathrm{t}$ d'atrazine et $8 \mathrm{t}$ de simazine employées à usage non agricole.

L'analyse des eaux des rivières, après chaque épisode pluvieux supérieur à $10 \mathrm{~mm}$ au cours des années 1990, 1991 et 1992, donne l'image d'une situation quelque peu préoccupante. En effet, sur une période allant d'avril à juillet pour chaque année du suivi, $90 \%$ des échantillons pour l'atrazine et $60 \%$ pour la simazine ont présenté des teneurs supérieures à $0,1 \mu \mathrm{g} . \mathrm{I}^{-1}$. Plus délicat encore, $50 \%$ des échantillons ont présenté des concentrations en atrazine supérieures à 1 $\mu \mathrm{g}^{-1}$. À ce stade, et sans prendre en compte les quelques cas où l'atrazine a atteint des concentrations de 10 et $15 \mu \mathrm{g} .\left.\right|^{-1}$, il devient technique- 
ment et économiquement difficile de respecter la concentration limite réglementaire de $0,1 \mu \mathrm{g} \mathrm{I}^{-1}$ pour les eaux de boisson (Gillet, 1992).

Ainsi, la pollution des eaux de surface se présente comme une pollution limitée dans le temps et étroitement corrélée aux épisodes pluvieux qui interviennent immédiatement après le traitement des sols ou des cultures. Pour la Bretagne, les maxima de concentration en atrazine ont été observés en juin et pour la simazine en mai.

Enfin, la comparaison des résultats obtenus pour ces 2 matières actives fait apparaître que la pollution des eaux de surface, dans son intensité et dans sa fréquence, n'est pas directement liée aux quantités utilisées. La pollution par les 2 herbicides est du même ordre bien que la simazine soit 50 fois moins utilisée que l'atrazine. Pour mieux appréhender les risques de pollution au cours de l'année, il s'avère nécessaire de connaître :

- le type d'usage qui est fait du pesticide ;

- la fréquence et l'ampleur des phénomènes de ruissellement en relation avec le climat, la topographie, le type de sol, le couvert végétal, le type de surface sur lequel il est appliqué et sa situation par rapport au cours d'eau ;

- le positionnement de ces ruissellements par rapport à la date d'emploi des produits et à leur durée de vie ;

- les interactions sol-produit-eau qui définiront la solubilisation de la matière active.

Le cas de chaque région est unique et s'intéresser à d'autres régions n'a pas pour but d'effectuer des comparaisons du degré de contamination de l'eau, mais de comprendre que chaque résultat est relatif et que chaque étude doit s'adapter aux particularités locales. Ainsi, les résultats présentés par Brambilla et al (1993) pour les eaux du Po en Italie (tableau II) présen-

Tableau II. Concentrations maximales en produits phytosanitaires observées au cours d'un suivi des eaux du Po allant de mai 1988 à juin 1991 (d'après Brambilla et al, 1993).

Produits recherchés $\begin{gathered}\text { Concentrations maximales } \\ \text { enregistrées }\end{gathered}$
ente

Atrazine

Dééthylatrazine

Simazine

Terbuthylazine
$0,30 \mu \mathrm{g} . \mathrm{I}^{-1}$ en mai 1989

$0,12 \mu \mathrm{g} . \mathrm{I}^{-1}$ en janvier 1990

$0,18 \mu \mathrm{g} . I^{-1}$ en mai 1989

$0,30 \mu \mathrm{g} . \mathrm{I}^{-1}$ en mai 1990 tent un intérêt, non pas parce qu'ils sont globalement plus satisfaisants par rapport à la Bretagne dans l'ampleur de la pollution par l'atrazine, mais parce qu'ils montrent que les métabolites des matières actives employées peuvent jouer un rôle important dans la pollution de l'eau.

De même, les résultats enregistrés par Maguire et Tkacz (1993) (tableau III) pour la rivière Yamaska (Québec), apparaissent comme particulièrement inquiétants, mais leur intérêt particulier est de nous montrer la diversité des produits pouvant être entraînés par ruissellement et l'amplitude des variations saisonnières.

\section{Le ruissellement à l'origine de la pollution des eaux de surface}

La réalisation d'un état des lieux à un instant donné n'a pas nécessairement pour objectif de donner une image exacte de la situation, mais de situer un degré de pollution. De même, il ne donne aucune information sur son évolution possible. Aussi, toute approche prévisionnelle ne peut être envisagée que par une connaissance plus fine des mécanismes de transfert par ruissellement.

Le transport latéral des pesticides à la surface de la parcelle jusqu'au cours d'eau est soumis à 2 facteurs limitants, liés d'une part à la topographie (nécessité d'une pente) et d'autre part au cli-

Tableau III. Pesticides dosés dans la rivière Yamaska (près du lac Saint-Pierre, Canada).

\begin{tabular}{lrrr} 
Pesticides & \multicolumn{3}{c}{1987} \\
\cline { 2 - 4 } & 9 juin & 8 juillet & 25 septembre \\
& & & \\
& & & \\
Atrazine & 845,0 & 37800,0 & 1690,0 \\
Chlorpyrifos & & 6,2 & \\
Dééthylatrazine & 4,9 & 86,2 & 131,5 \\
Diazinon & & 11,9 & 2,1 \\
Dichlorvos & & & 1,7 \\
Endrin & & 0,8 & 0,6 \\
Heptachlor & & 2,1 & 1,7 \\
Heptachlor époxyde & 1,8 & 5,6 & 0,3 \\
Lindane & 0,3 & 7,6 & 2,9 \\
Métolachlore & 103,7 & 4660,0 & 71,0 \\
Naled & 5,5 & 74,8 & 52,7 \\
Simazine & 1,8 & 31,6 & 40,9 \\
& & &
\end{tabular}

Résultats exprimés en ng..$^{-1}$ (d'après Maguire et Tkacz, 1993). 
mat (intervention de précipitations plus ou moins intenses et donnant lieu ou non à des transports de sédiments). II est par ailleurs dépendant de la solubilité des produits dans l'eau et de la stabilité de leurs liaisons avec les constituants du sol. Le développement des surfaces érosives est luimême contrôlé par les caractéristiques du sol : texture, stabilité structurale, façons culturales et couvert végétal.

De nombreux travaux ont été réalisés en vue d'évaluer les quantités de résidus transportés par ruissellement, voire par érosion. Ils ont été menés aussi bien en conditions expérimentales naturelles ou semi-naturelles (pluies simulées) que modélisées (précipitations simulées, pente contrôlée...). Ces 3 approches sont complémentaires et conduisent à de nombreuses conclusions communes.

Le transport latéral des résidus est essentiellement réalisé sous forme hydrosoluble, en général 7 à 10 fois plus que par les sédiments, même si la concentration des pesticides dans ceux-ci est toujours de 2 à 15 fois plus forte que dans l'eau (White et al, 1967 ; Hall et al, 1972 ; Hall, 1974 ; Ritter et al, 1974 ; Leonard et al, 1979). II faut, par ailleurs, noter l'absence de relation entre les quantités de sédiments entraînés et de résidus exportés (Wauchope, 1987). Ceci s'explique par les forts volumes d'eau mis en jeu par rapport aux faibles masses de sol déplacées.

En termes de quantités de produits transportés, Ritter et al (1974) observent que les transferts sont nuls si les précipitations donnant lieu à un ruissellement se produisent plus de 2 mois après application du produit phytosanitaire. Ceci paraît en contradiction avec les observations de Wu (1980) qui enregistre pour le même herbicide, des traces dans les eaux même en fin de saison.

Globalement, et dans des conditions particulièrement favorables au ruissellement, les exportations (tableau IV) sont généralement comprises entre 0,1 et $3 \%$ et n'excèdent pas $12 \%$ de la dose appliquée. Toutefois, Ritter et al (1974) ont obtenu en 1970 pour l'atrazine des exportations atteignant $15,9 \%$.

Même si les quantités transférées sont le plus souvent inférieures à $50 \mathrm{~g} \cdot \mathrm{ha}^{-1} \cdot \mathrm{an}^{-1}$ pour des traitements du sol aux doses préconisées par le fabricant, la concentration en résidus des eaux de ruissellement peut être ponctuellement très élevée (tableau $\mathrm{V}$ ) et atteindre des valeurs de l'ordre de $8,8 \mathrm{mg}^{-1} \mathrm{I}^{-1}$ (Triplett et al, 1978).

Tous les auteurs notent les plus fortes teneurs en résidus dans les premières eaux de ruissellement obtenues après traitement. D'après

Tableau IV. Exportations totales de pesticides par ruissellement mesurées par différents auteurs sous conditions de plein champ.

\begin{tabular}{|c|c|c|c|c|}
\hline $\begin{array}{l}\text { Produit et } \\
\text { dose }\left(\mathrm{kg}^{\mathrm{h}} \mathrm{ha}^{-1}\right)\end{array}$ & $\begin{array}{l}\text { Durée du } \\
\text { suivi (ans) }\end{array}$ & $\begin{array}{l}\text { Pente du } \\
\text { sol }(\%)\end{array}$ & $\begin{array}{c}\text { Pertes a } \\
\left(^{\text {a }}\right)^{*}\end{array}$ & Auteurs \\
\hline Alachlore $(1,35)$ & 1 & - & 11,6 & Paterson et Schnoor, 1992 \\
\hline Alachlore $(2,3)$ & 1 & - & 0,16 & Wu, 1980 \\
\hline Amétryne $(4,5)$ & 1 & $10-12$ & 7,7 & Liu et al, 1985 \\
\hline Atrazine $(0,78)$ & 1 & - & 10,2 & Paterson et Schnoor, 1992 \\
\hline Atrazine $(1,7)$ & 1 & 5 & 1 & Wu, 1980 \\
\hline Atrazine $(1,5-4)$ & 4 & $4-10$ & $0,2-0,9$ & Leonard et al, 1979 \\
\hline Atrazine $(1,7)$ & 1 & - & 1,7 & Triplet et al, 1978 \\
\hline Atrazine $(3,4)$ & 1 & - & 5,4 & Ritter et al, 1974 \\
\hline Cyanazine $(1,3-1,6)$ & 1 & $4-10$ & $0,07-1$ & Leonard et al, 1979 \\
\hline Difénamide $(2,3-3,5)$ & 4 & $3-8$ & $0,1-7,2$ & \\
\hline Diuron $(3,4)$ & 1 & - & 5,6 & Liu et al, 1985 \\
\hline Néburon $(1,6)$ & 1 & $5-7$ & 0,04 & Copin et Deleu, 1985 \\
\hline Nitrofène $(0,5)$ & 1 & - & 0,05 & \\
\hline Propachlore $(6,8)$ & 1 & $10-15$ & 3,1 & Ritter et al, 1974 \\
\hline Propazine $(1,7)$ & 1 & $3-8$ & 6,7 & Leonard et al, 1979 \\
\hline Simazine $(1,7)$ & 1 & - & 2,1 & Triplet et al, 1978 \\
\hline Trifluraline $(1,1)$ & 2 & $3-8$ & $0,1-0,3$ & Leonard et al, 1979 \\
\hline
\end{tabular}

\footnotetext{
*\% de la dose appliquée.
} 
Tableau V. Niveaux de concentration pour différents pesticides entraînés par ruissellement.

\begin{tabular}{|c|c|c|c|c|}
\hline \multirow{2}{*}{$\begin{array}{l}\text { Produit et } \\
\text { dose }\left(\mathrm{kg}^{\mathrm{h}} \mathrm{ha}^{-1}\right)\end{array}$} & \multirow{2}{*}{$\begin{array}{l}\text { Solubilité a } \\
\text { dans l'eau }\end{array}$} & \multicolumn{2}{|c|}{ Niveaux de concentration } & \multirow[t]{2}{*}{ Auteurs } \\
\hline & & $\operatorname{Eau}\left(\mu g . \digamma^{-1}\right)$ & Sédiments (mg.kg-1) & \\
\hline Amétryne $(4,5)$ & 185 & $10-250$ & $0,14-1,13$ & Liu et al, 1985 \\
\hline Atrazine $(1,5-4)$ & 28 & $0,2-1900$ & $0,06-4,1$ & Leonard et al, 1979 \\
\hline Cyanazine $(1,3-1,6)$ & 171 & $2-180$ & $0,1-2,3$ & \\
\hline Difénamide $(2,3-3,5)$ & 260 & $0,3-2070$ & $0,7-1,7$ & \\
\hline Diuron $(3,4)$ & 42 & $10-450$ & $0,2-5,8$ & Liu et al, 1985 \\
\hline Propazine $(1,7)$ & 8,6 & $16-400$ & $0-0,9$ & Leonard et al, 1979 \\
\hline Trifluraline $(1,1)$ & 1 & $0,1-21$ & $0,01-0,3$ & \\
\hline \multicolumn{5}{|c|}{ eau + sédiments $\left(\mu g . \digamma^{-1}\right)$} \\
\hline Alachlore & 242 & \multicolumn{2}{|c|}{$0-370$} & Paterson et Schnoor, 1992 \\
\hline Alachlore & 242 & \multicolumn{2}{|c|}{$<2,9$} & Wu, 1980 \\
\hline Atrazine & 28 & \multicolumn{2}{|c|}{$0-200$} & Paterson et Schnoor, 1992 \\
\hline Atrazine & 28 & \multicolumn{2}{|c|}{$0,08-52$} & Wu, 1980 \\
\hline Atrazine & 28 & \multicolumn{2}{|c|}{$100-480$} & Triplet et al, 1978 \\
\hline Simazine & 3,5 & \multicolumn{2}{|c|}{$50-1200$} & Triplet et al, 1978 \\
\hline
\end{tabular}

a en mg.t-1 d'après The Agrochemicals Handbook - Royal Society of Chemistry.

Léonard et al (1979), cette concentration décroît exponentiellement avec le temps et dépend des quantités de résidus présentes à la surface du sol dans la zone explorée par les eaux de ruissellement. Les travaux de White et al (1967) montrent que la concentration en atrazine des eaux de ruissellement diminue au cours d'une phase d'écoulement et pour les écoulement successifs.

Si la quantité, la fréquence et la force des précipitations constituent 3 paramètres importants de la régulation des pertes de produits phytosanitaires par ruissellement, il faut également prendre en compte le sol et son mode de travail, la technique d'application des produits, ainsi que le couvert végétal (Hall et al, 1983). Pour Triplett et al (1978), le travail du sol ne joue qu'un rôle minime, mais Sauer et Daniel (1987) observent des pertes significativement plus faibles en atrazine et alachlore pour des sols travaillés suivant des méthodes classiques (labour) par rapport aux techniques utilisées en vue de la protection du sol contre l'érosion (tableau VI). Les auteurs attribuent ce résultat à la présence des résidus de récolte à la surface des sols soumis au travail minimum. Ces résidus peuvent intercepter l'herbicide lors du traitement, et celui-ci est ensuite facilement lessivé ou entraîné par les eaux de ruissellement. Les résultats de Sauer et Daniel (1987) sont toutefois en contradiction avec ceux
Tableau VI. Exportations en atrazine et alachlore par les eaux de ruissellement pour différents types de travail du sol et époques (d'après Sauer et Daniel, 1987).

Travail du sol

Pertes en g.ha-1

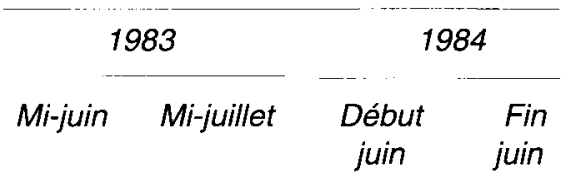

\begin{tabular}{lrrrr} 
& \multicolumn{4}{c}{ Atrazine } \\
Conventionnel & 5,7 & 2,4 & 117,0 & 8,8 \\
Chisel & 5,5 & 1,9 & 82,4 & 7,8 \\
Billons & 7,8 & 1,4 & 239,0 & 10,3 \\
Non travail & 8,1 & 2,0 & 202,0 & 9,7 \\
\multicolumn{4}{c}{ Alachlore } \\
Conventionnel & 7,2 & 0,4 & 82,3 & 3,9 \\
Chisel & 6,6 & 0,2 & 58,2 & 3,1 \\
Billons & 9,9 & 0,2 & 166,0 & 2,7 \\
Non travail & 8,8 & 0,2 & 133,0 & 2,9 \\
& & & &
\end{tabular}

de Kenimers et al (1989), pour qui la matière organique présente à la surface du sol jouerait un rôle protecteur vis-à-vis des transferts par ruissellement (tableau VII). Cependant, il est difficile d'évaluer en toutes circonstances les effets des paramètres cités, dans la mesure où les auteurs ne précisent pas la forme des résidus 
Tableau VII. Tranferts latéraux d'atrazine mesurés $24 \mathrm{~h}$ après traitement, sur des mini-parcelles traitées à la dose de $2,24 \mathrm{~kg} \cdot \mathrm{ha}^{-1}$. Effets du travail du sol et de la matière organique à sa surface (d'après Kenimers et al, 1989).

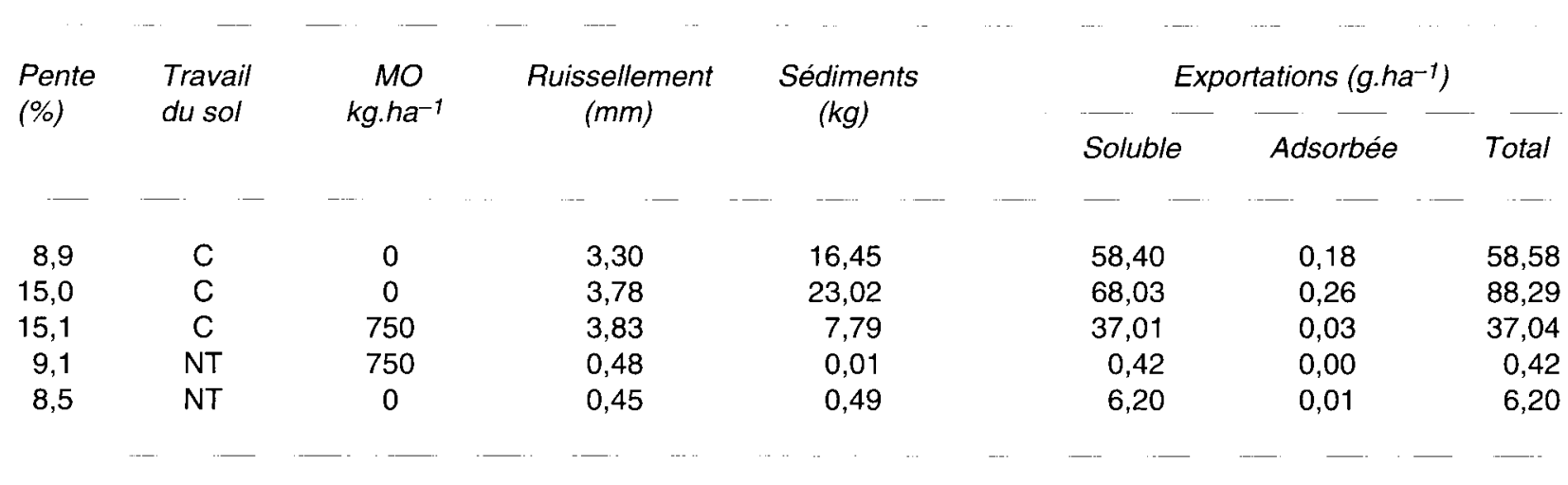

$C:$ travail conventionnel ; NT : non-travail ; $M O$ : matière organique.

dosés : forme dissoute et entraînée par l'eau, forme adsorbée et véhiculée par les particules érodées. Cette seconde voie de transfert étant prépondérante pour les pesticides fortement adsorbés par les constituants du sol.

La végétation, par sa densité et son stade de développement, modifie également les mouvements latéraux. En effet, la densité de semis et l'évapotranspiration dans une certaine mesure, influent sur le nombre et le volume des ruissellements, en particulier à partir du milieu du cycle de la culture (Hall et al, 1972).

Au total, les pertes par ruissellement et érosion sont gouvernées principalement par le volume et l'intensité des précipitations qui interviennent à un moment proche du traitement. Elles sont également dépendantes de la dose, de la formulation, du mode d'application, de la persistance et de la rémanence de la matière active, du type de culture et de sa densité, de la topographie, des caractéristiques physiques du sol (stabilité structurale, humidité au moment des nouvelles précipitations), et des techniques culturales.

II est possible d'intervenir sur certains de ces paramètres afin de maîtriser les transferts des pesticides dans les eaux de surface sans pour autant réduire les performances des traitements. II reste toutefois à en préciser les modalités. La mise en place de bandes enherbées en bordure des cours d'eaux constitue à cet égard une technique qui connaît actuellement un certain intérêt (Réal et al, 1993).

À la pollution des eaux de surface par les eaux de ruissellement, il faut ajouter celle due aux eaux de drainage pour tous les secteurs où l'assainissement des sols a été entrepris. Leur contribution sera examinée dans le cadre de la pollution des eaux souterraines, dans la mesure où leur contamination renseigne sur la qualité des eaux susceptibles de migrer vers la nappe.

\section{NIVEAU DE CONTAMINATION DES EAUX SOUTERRAINES}

\section{Cas de la Lorraine}

Si le niveau de pollution des eaux de surface est défini en quelque sorte par l'efficience de l'eau de ruissellement à transférer les produits phytosanitaires, en revanche, la pollution des eaux souterraines dépend du processus de lessivage, mécanisme plus lent et qui présuppose un rôle important du sol en tant que filtre biologique.

Une action importante a été menée en Lorraine pour établir un état des lieux sur cet aspect : 173 captages souterrains, alimentant en eau potable des villes de 2000 à 10000 habitants (sauf dans les Vosges où la limite est plus basse), ont été examinés à l'initiative des ministères de l'Agriculture, de l'Environnement et de la Santé, représentés par les instances locales. En fonction des pratiques agricoles, 17 produits ont été recherchés, sans connaître cependant les quantités réelles utilisées. Les prélèvements ont été effectués dans le courant du mois de juillet 1991, indépendamment des différentes dates d'emploi des matières actives (Chambre d'agriculture de Lorraine, 1992).

Parmi les 17 substances recherchées, 5 sont apparues à des fréquences variables. II s'agit de l'atrazine, la simazine, le lindane, le carbofuran et l'isoproturon (pour ce produit il s'agirait en fait de diverses phénylurées). L'atrazine était présente dans $55 \%$ des échantillons, la simazine dans 
$20 \%$, le lindane dans $40 \%$. Environ $50 \%$ des échantillons étaient contaminés par au moins 2 produits et moins de $10 \%$ contenaient 3 matières actives. En termes de concentration, $53 \%$ des captages dépassaient à cette date $0,1 \mu \mathrm{g} . \mathrm{I}^{-1}$ pour une substance, en général l'atrazine, mais aussi quelques cas pour la simazine et le carbofuran. Seulement $5 \%$ des échantillons ont présenté des teneurs supérieures à $0,5 \mu \mathrm{g} . \mathrm{I}^{-1}$.

Sans porter de jugement sur la gravité de la situation en Lorraine, on se limitera à souligner que sa position par rapport à la Lombardie par exemple (tableau VIII), peut être due à un ensemble de facteurs qui différencient ces 2 régions : occupation des sols, doses appliquées, nature du sol et du sous-sol, activité biologique et vitesse de dégradation des produits, rythme et intensité des précipitations.

Les résultats examinés pour la Lorraine ne concernent qu'un seul prélèvement pour chaque site et, à ce titre, il est logique de s'interroger sur la signification de ces résultats et en particulier sur leur évolution dans le temps. Le suivi hebdomadaire d'un point de captage en Lorraine a permis à Portal et al (1992) de mettre en évidence des variations du niveau de contamination dans le temps. Les résultats présentés par la figure 1 montrent une certaine stabilité de la concentration en atrazine. Pour le cas présenté, on peut estimer que l'enrichissement en résidus s-triaziniques des nappes situées en zone de culture de maïs s'est réalisé régulièrement depuis 2025 ans en Lorraine et que l'eau captée actuellement traduit la mise en place d'un état "d'équilibre". II n'en est pas nécessairement de même pour des produits d'usage moins régulier, plus récents et plus dégradables tel l'isoproturon, qui apparaît au niveau de la nappe sans relation évidente avec la pluviométrie ou avec la date d'application. Ici, le dosage hebdomadaire de l'état de pollution peut donner une réponse du type «tout ou rien», même pour la période où le

Tableau VIII. Pollution par l'atrazine de l'aquifère de Lombardie (d'après Donati et al, 1993).

Sources Nombre Classes de concentrations ( $\left.\mu g . \digamma^{-1}\right)$ analysées

\begin{tabular}{lrrrrr} 
& & & & $\begin{array}{c}0,1 \\
(\%)\end{array}$ & $>1$ \\
\hline & $\ldots$ & $\ldots$ & - & - \\
Publiques & 2005 & & 84,5 & 14,0 & 1,4 \\
Privées & 956 & 37,5 & 56,1 & 6,4
\end{tabular}

niveau de contamination exprimé par la moyenne mensuelle est élevé, ce qui n'est pas satisfaisant du point de vue du contrôle sanitaire (Portal et al, 1994). Enfin, on remarquera la forte contamination par la dé-éthylatrazine, avec des fluctuations importantes, vraisemblablement liées à des phases d'activité biologique intense à l'origine de la biodégradation de l'atrazine à la surface du sol. Ainsi, pour certains produits phytosanitaires, ce n'est pas la dispersion de la seule matière active qui doit être considérée mais également celle de ses métabolites majeurs.

Comme pour les eaux de surface, ici encore, pour établir un jugement prédictif, il est indispensable d'effectuer une analyse des mécanismes de transfert par lessivage grâce à des dispositifs simples prenant en compte, en particulier, le rôle du climat et celui spécifique, des différents types de sol.

\section{Le lessivage à l'origine de la pollution des eaux souterraines}

L'étude du mouvement vertical a fait l'objet de nombreux travaux, menés en faisant appel aussi bien à la parcelle drainée qu'au modèle de plein champ (Boesten, 1987). Seul un nombre limité de résultats sera rapporté ici.

\section{Transfert dans les couches supérieures des sols drainés}

Les concentrations en résidus enregistrées dans les eaux de drainage, susceptibles de représenter celles des eaux qui vont pour partie s'écouler vers la nappe, sont très variables (tableau IX). Elles fluctuent entre 0,01 et $29 \mu \mathrm{g} . \mathrm{l}^{-1}$. Ces concentrations diminuent avec le temps qui sépare le traitement de la phase de drainage. Les premières eaux d'une même phase sont les plus riches en résidus (Schiavon et Jacquin, 1973).

Tout aussi variables sont les évaluations des quantités exportées (tableau IX). Cette variabilité est étroitement liée au type de sol, à la topographie, à la nature et à la distribution des précipitations au cours de l'année.

L'utilisation de colonnes de sol ou de cases lysimétriques pour l'étude du mouvement vertical des pesticides est relativement courante (Jarczyk, 1983, 1987 ; Albanis et al, 1988). Ce mode d'investigation présente au moins 2 avantages : d'une part, il fournit des résultats qui intègrent, comme au niveau de la parcelle, les effets 


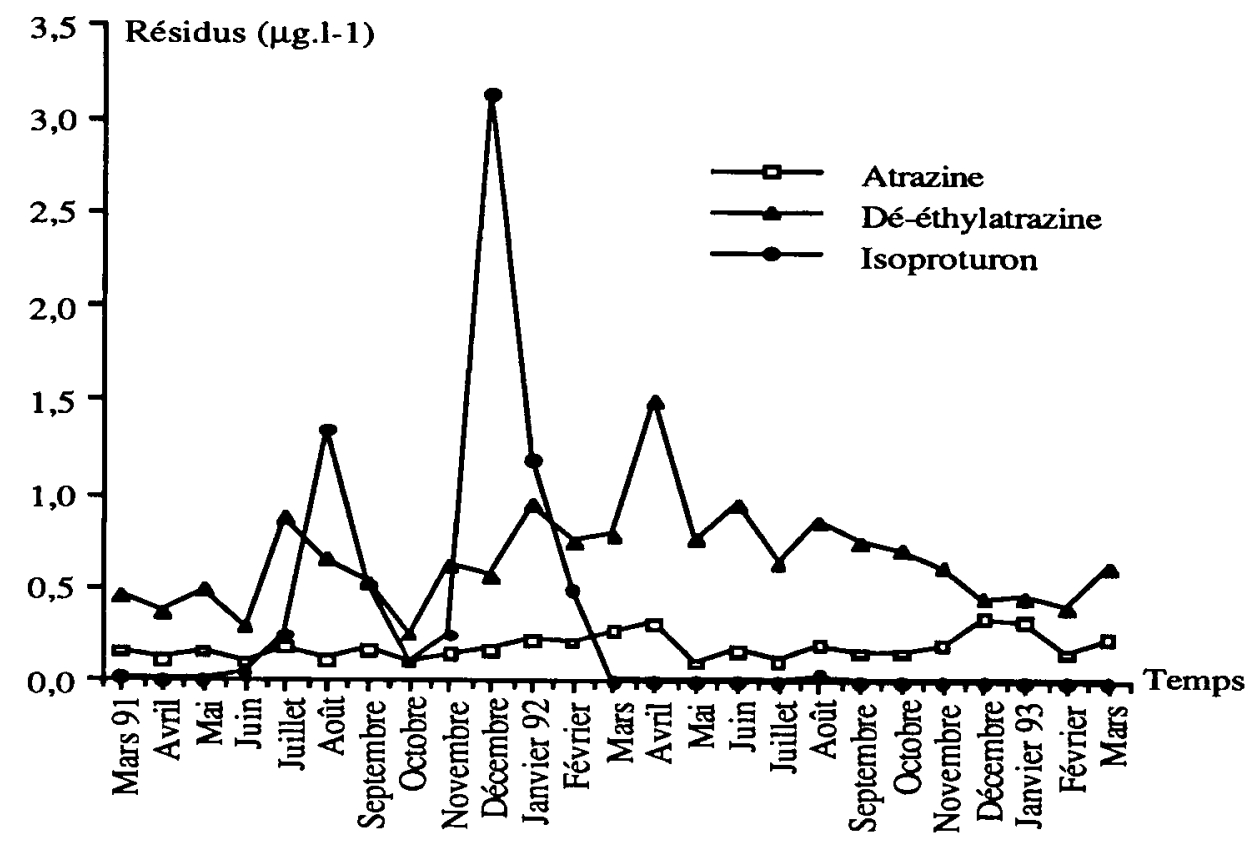

Fig 1. Évolution des teneurs en atrazine, dé-éthylatrazine et isoproturon de l'eau d'un captage de Lorraine situé sur calcaire du Bajocien à $5 \mathrm{~m}$ de profondeur (d'après Portal et al, 1992). des conditions climatiques, d'autre part, il permet l'utilisation de molécules marquées au ${ }^{14} \mathrm{C}$ et donc l'identification et la quantification plus précise des produits issus de la dégradation de la molécule mère (Kubiak et al, 1988). S'agissant de l'atrazine, les valeurs obtenues pour l'année 1981-1982 avec un sol limoneux $(850 \mathrm{~mm}$ de précipitations, $1,6 \mathrm{~kg} \cdot \mathrm{ha}^{-1}$ ) ont montré un entraînement par les eaux de percolation de $5,6 \%$ de la radioactivité appliquée (Schiavon, 1988), dans lequel l'atrazine ne représente qu'environ $10 \%$ (fig 2) ; le reste étant constitué par l'ensemble des produits issus de sa dégradation. Cette valeur correspond sensiblement au pourcentage d'atrazine exportée par le drainage de ce même sol en 1972, et montre bien que dans la pollution des nappes phréatiques l'atrazine peut, dans certains cas, être minoritaire. De plus, cette valeur est très proche de celles obtenues par Albanis et al (1988) avec des cases lysimétriques et différents types de sol : après $1324 \mathrm{~mm}$ de précipitations, $0,54,0,66$ et $0,47 \%$ de la dose appliquée sont lessivés, respectivement pour un sol argileux, limoneux et limono-sableux.

Tableau IX. Variations des teneurs en pesticides des eaux de drainage et quantités exportées.

\section{Produits et dose $\left(\mathrm{kg}_{\mathrm{h}} \mathrm{ha}^{-1}\right)$}

Atrazine $(1,25)$

Atrazine $(1,25)$

Atrazine $(1,25)$

Atrazine $(1,25)$

MCPA (2)

MCPA (2)

Dichlorprop (2)

Dichlorprop (2)

Atrazine $(1,6)$

Métolachlore $(2,6)$

Chlortoluron $(2,1)$

Chlortoluron $(2,1)$

Isoproturon $(2,1)$

Isoproturon $(2,1)$

\section{Nature \\ des sols}

Argileux

Limoneux

Argileux

Limoneux

Argileux

Sableux

Argileux

Sableux

Argilo-limoneux

Argilo-limoneux

Argileux

Limoneux

Argileux

Limoneux

\section{Concentration \\ $\left(\mu g . \vdash^{1}\right)$}

\section{$0,5-28$}

$0,5-24$

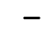

$-$

0,05

$1-15$

$0,1-0,6$

$1-23$

$0,01-3,5$

$1,9-29,3$

2-11

1,5-4,1

$0,01-3,4$

$1,7-2,5$

$\begin{gathered}\text { Exporté } \\ (\%)\end{gathered}$

$1,94^{\star}$
$0,54^{\star}$
$0,85^{\circ}$
$0,005^{\circ}$
$>0$
0,4
0,06
0,9
0,04
$>0$
0,28
0,22
0,03
0,03

\section{Auteurs}

Schiavon et Jacquin, 1973

Schiavon et Jacquin, 1973

Schiavon, 1980

Schiavon, 1980

Kreuger et Brink, 1988

Kreuger et Brink, 1988

Kreuger et Brink, 1988

Kreuger et Brink, 1988

Southwick et al, 1990

Southwick et al, 1990

Schiavon et al, 1992

Schiavon et al, 1992

Schiavon et al, 1992

Schiavon et al, 1992

\footnotetext{
* : valeurs de l'année $1972-19733^{\circ}$ valeurs de l'année 1973-1974.
} 


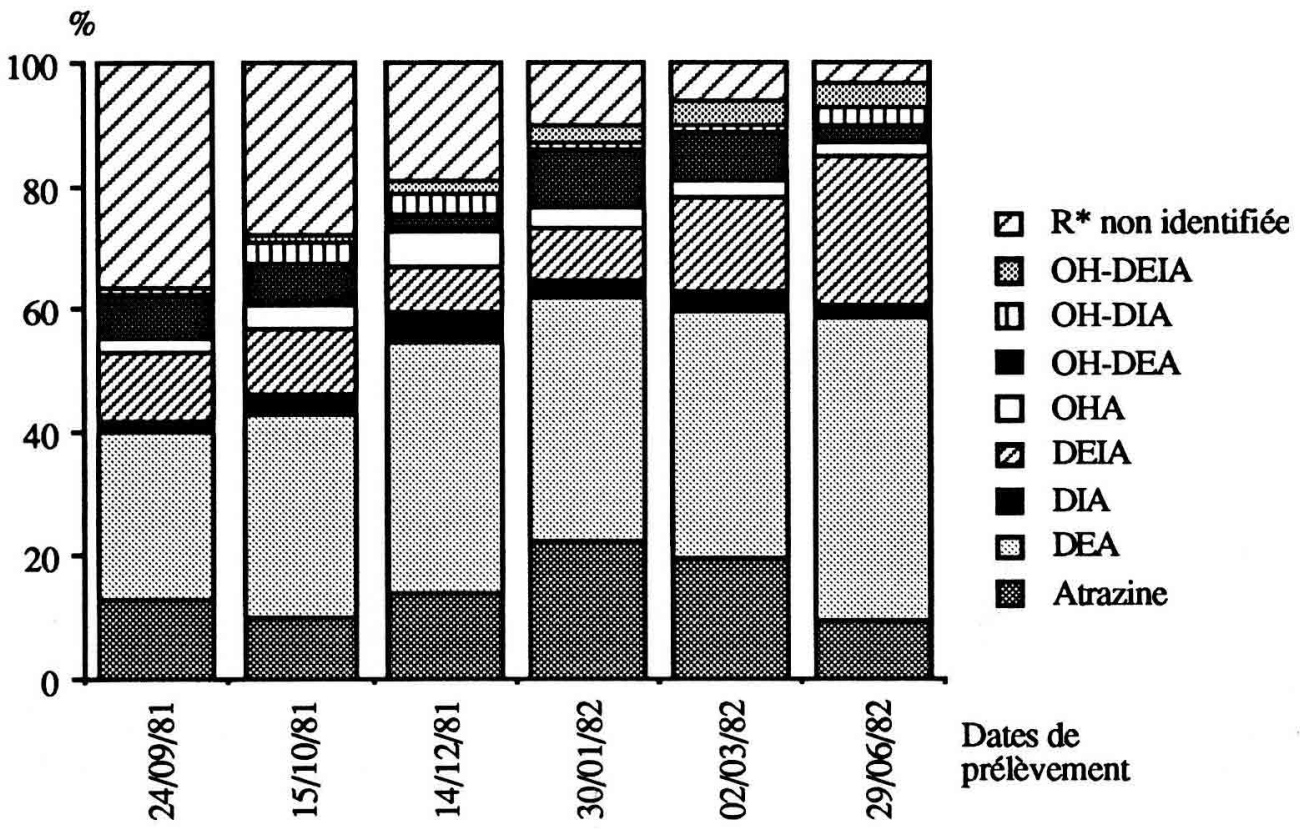

Fig 2. Composition des eaux de percolation de colonnes de sol traitées 05/1981) avec de l'atrazine marquée au ${ }^{14} \mathrm{C}$ sur le noyau en fonction des dates de prélèvement et exprimée en pourcentage de la radioactivité présente dans chaque échantillon (d'après Schiavon, 1988). DEA dé-éthylatrazine, DIA dé-isotpropylatrazine, DEIA : dé-éthyl, dé-isopropylatrazine, OHA : hydroxyatrazine, $\mathrm{OH}$ DEA : 2-hydroxy, dééthylatrazine, $\mathrm{OH}$-DIA 2-hydroxy-dé-isopropylatrazine, OH-DEIA : 2hydroxy, dé-éthyl, dé-isopropylatrazine, $\mathrm{R}^{*}$ radioactivité.

Ainsi, ces techniques permettent d'accéder d'une manière plus aisée à des résultats proches de ceux obtenus au niveau de la parcelle drainée. Par ailleurs, l'emploi de molécules marquées met en évidence l'effort analytique qui doit être réalisé pour évaluer, au niveau de la pratique courante, la pollution réelle du sol et des nappes par les pesticides et leurs produits de dégradation.

\section{Transfert dans le sous-sol}

Étant donné les faibles possibilités de réadsorption des pesticides par les couches profondes du sol et la dégradation limitée (Dictor, 1994 ; Kruger et al, 1993), on peut estimer que les concentrations en résidus des eaux circulant dans ces couches sont proches de celles se dirigeant vers la nappe. Ainsi, par le suivi de la teneur en résidus des eaux de drainage et l'établissement d'un bilan hydrique, il est possible d'apprécier les transferts vers les nappes. Si les quantités transférées par lessivage sont fonction des propriétés bio-physicochimiques de la couche de labour et des conditions climatiques, en revanche, la vitesse de progression vers la nappe dépend essentiellement de la pluviométrie.

Mais l'évolution des possibilités de pollution des eaux souterraines doit tenir compte du rôle particulier joué par le sol en tant que système de stockage des produits phytosanitaires et de leur métabolites. II faut donc prendre en compte son état de pollution et sa propre évolution possible.

\section{Incidence de la pollution du sol}

La pollution des sols doit être considérée sous 2 aspects. La première est une pollution mesurable, au niveau de la parcelle traitée, par les techniques classiques d'extraction et d'analyse (chromatographie en phase gazeuse ou liquide). La seconde est une pollution discrète, qui a été mise en évidence à l'aide de molécules marquées et qui est associée à la formation de liaisons temporairement stables entre le pesticide et/ou ses métabolites et les constituants du sol. Outre l'utilisation d'une molécule marquée, le suivi de cette pollution discrète ne peut être réalisé qu'à l'aide d'un système simple de type lysimètre ou colonne de sol, placé sous conditions naturelles, et permettant de réaliser un bilan (Perrin-Ganier et al, 1994).

Les travaux menés avec des produits très divers par leur nature chimique tendent à montrer que le phénomène intéresse toutes les familles d'herbicides (tableau $X$ ) et plus généralement, tous les produits phytosanitaires. L'ampleur du phénomène dépend de la réactivité chimique de la matière active et s'exprime avec le maximum d'intensité dans les premiers centimètres de la couche de labour, riche en matière organique et en résidus. Ce type de réaction suppose la perte de l'identité chimique du produit lié et sa possibilité de polymérisation avec les macromolécules organiques (Bartha, 1971). En fait, les résidus non extractibles constituent un ensemble hétérogène à la fois par leur nature chimique (matière active et produits de dégradation) et leur mode 
Tableau X. Exemples de formation de résidus non extractibles (RNE) dans les sols sous conditions naturelles.

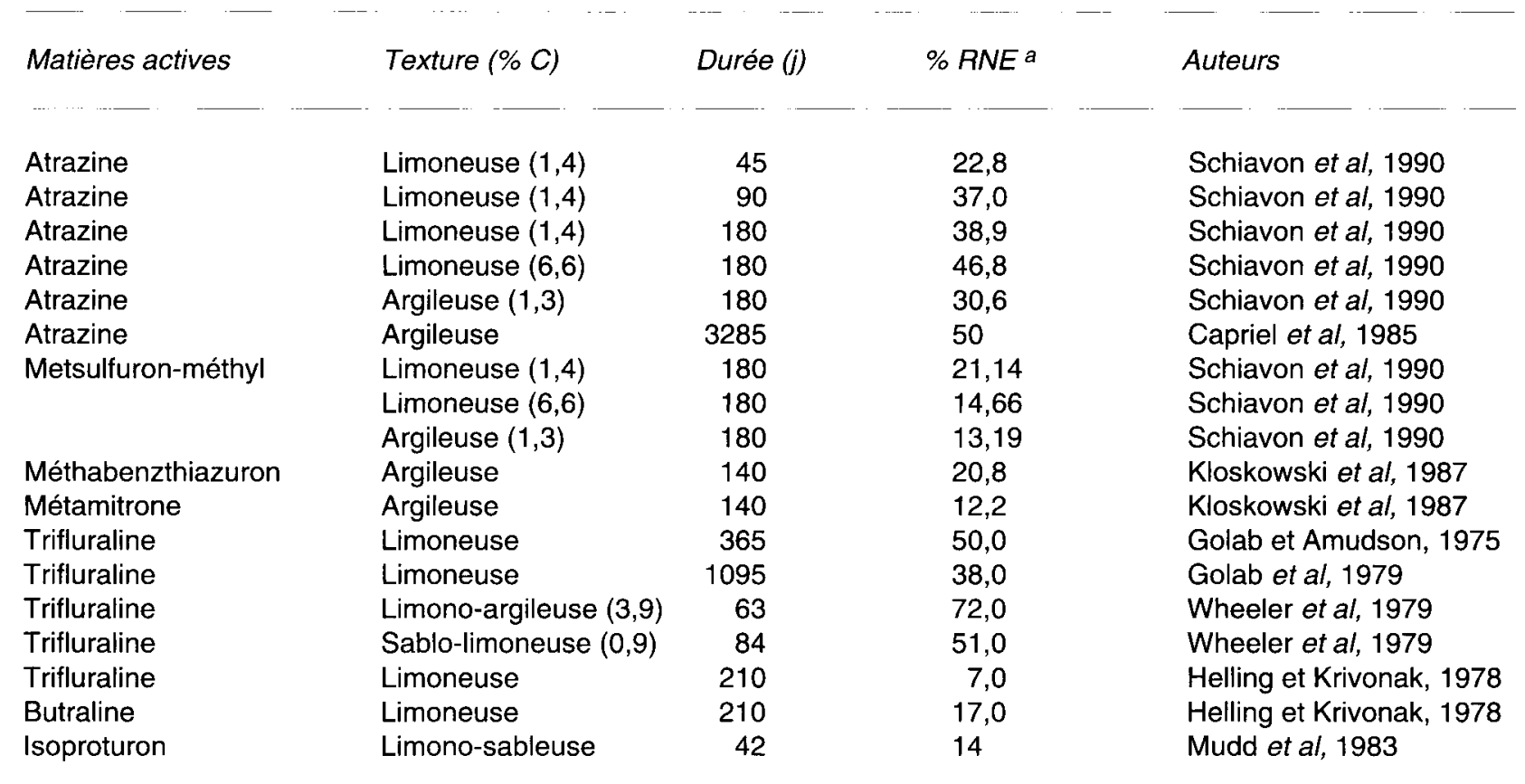

a \% de la dose appliquée

de rétention (liaison chimique et/ou emprisonnement dans le réseau tridimensionnel de la matière organique, dans les espaces interfoliaires des argiles ou dans la microporosité du sol).

Cette immobilisation des résidus par les constituants du sol pourrait être considérée comme un processus contribuant à réduire les risques de pollution de l'eau. II s'avère cependant qu'une partie d'entre eux au moins peut être remobilisée à la faveur des réorganisations des matières organiques (tableau $\mathrm{XI}$ ). Une fraction des résidus déstabilisés peut échapper à la minéralisation et de ce fait participer ultérieurement sous forme diffuse à la pollution de l'eau ou des végétaux (Bertin et al, 1990 ; Demon, 1994).

Le dosage des résidus dans les couches profondes est très rarement réalisé, mais il permet d'évaluer le stock de résidus disponibles pour un transfert vers la nappe. En effet, les résidus présents dans le sous-sol (tableau XII) pauvre en matière organique sont faiblement retenus (Calvet, 1989) et soumis à des processus de dégradation très lents (Cohen et al, 1984). Ils

Tableau XI. Évolution en conditions naturelles des résidus non extractibles dans 3 sols traités à l'atrazine.

Temps après traitement (j)

0
45
90
180
365

Résidus non extractibles (\%)

$\begin{array}{lcc}\text { Sol argileux } & \text { Sol limoneux } & \text { Sol calcaire } \\ (2,2 \% \mathrm{MO}) & (2,4 \% \mathrm{MO}) & (11,4 \% \mathrm{MO})\end{array}$

$\begin{array}{ccc}0 & 11,9 & 2,3 \\ 15,6 & 22,8 & 26,9 \\ 23,9 & 37,0 & 47,2 \\ 30,6 & 38,9 & 46,8 \\ 18,4 & 14,9 & 33,0\end{array}$

Résultats exprimés en pourcentage de la dose appliquée (d'après Schiavon et al, 1990). MO : matière organique. 
Tableau XII. Profondeurs atteintes par quelques pesticides à des teneurs dosables pour des sols de type sablolimoneux et des doses appliquées sensiblement identiques.

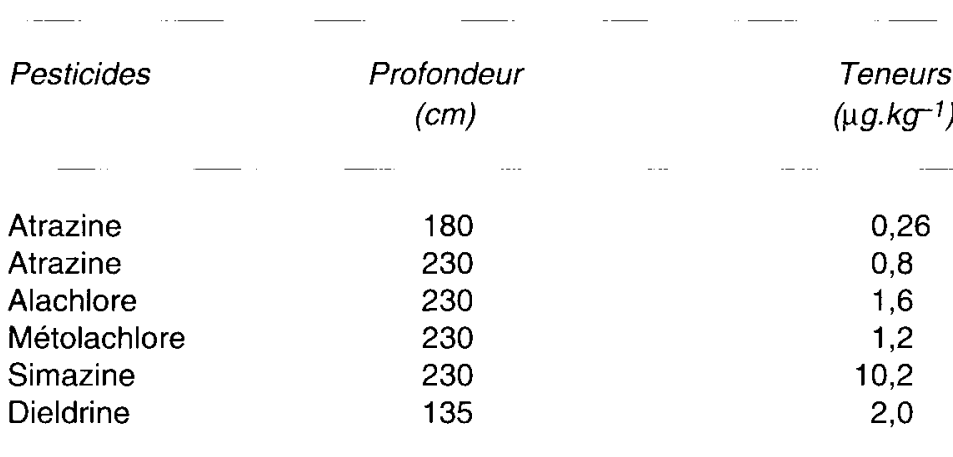

Auteurs
Wehtje et al, 1983
Huang et Frink, 1989 Huang et Frink, 1989 Huang et Frink, 1989 Huang et Frink, 1989 Halleberg, 1989

peuvent donc éventuellement constituer une réserve pour un transport inéluctable vers la nappe, régulé par la fréquence et la nature des précipitations (Bailey et White, 1970).

Vraisemblablement du fait des difficultés techniques d'échantillonnage en profondeur, les mesures réalisées sont peu nombreuses. Toutefois, elles tendent à montrer, d'une manière générale, de faibles progressions des pesticides vers la profondeur et des contaminations du sol réduites qui s'écartent de la réalité. En effet, l'utilisation des matières actives marquées au ${ }^{14} \mathrm{C}$ a permis de mettre en évidence qu'au cours du temps une partie des résidus devient progressivement non extractible et donc échappe au dosage classique. Ainsi la pollution réelle des sols est très souvent supérieure à celle mesurée.

Au total, le sol apparaît comme un système complexe qui contribue, par ses activités propices à la dégradation biologique ou abiotique, à réduire les risques de pollution de l'eau qui circule en son sein. Mais, dans la mesure où ses possibilités de dégradation sont limitées, il est impératif de vérifier que l'on n'assiste pas a un stockage de résidus à disponibilité variable, résidus qui pourraient entretenir une contamination quasi permanente de l'eau souterraine, qui serait facilitée dans certains cas par des interactions avec des composés organiques endogènes hydrosolubles (Madhun et al, 1986).

\section{CONCLUSION}

Les campagnes d'analyses des eaux de surface et souterraines lancées ces dernières années montrent d'une manière évidente que l'eau destinée à la consommation humaine est fréquemment polluée par certains produits phytosanitaires, parfois très au-delà du seuil de potabilité.
C'est une situation qui, plus ou moins aiguë, peut se rencontrer dans plusieurs périmètres soumis à l'agriculture intensive, mais il paraît certain que les utilisations non agricoles des pesticides jouent également un rôle non négligeable. Les études des mécanismes de transfert qui alimentent cette contamination montrent qu'il est possible d'agir sur certains paramètres. Ainsi, avant même de compléter les données pour alimenter des modèles mathématiques prédictifs de l'évolution de la contamination, il s'avère urgent d'identifier les pratiques culturales à mettre en place et susceptibles de la réduire. L'amélioration peut être rapide pour les eaux de surface, mais bien plus lente pour les eaux souterraines pour qui la pollution du sol entretiendra pendant un certain temps une pollution très diffuse.

\section{RÉFÉRENCES}

Albanis TA, Pomonis PJ, Sdoukos AT (1988) Movement of methyl parathion, lindane and atrazine through lysimeters in field conditions. Toxicol Environ Chemistry 17, 35-45

Bailey GW, White JL (1970) Factors influencing the adsorption, desorption and movement of pesticides in soil. Res Rev 32, 29-92

Barriuso E, Gaillardon P, Schiavon M (1994) Biodisponibilité des pesticides présents dans le sol. XXIVe Congrès du groupe français des pesticides, Bordeaux 18-19 mai 1994, 1-11

Bartha R (1971) Fate of herbicide-derived chloroanilines in soil. J Agr Food Chem 2, 385-387

Bertin G, Schiavon M, Pottier C (1990) Plant bioavailability of "natural" and "model" humic acid-bound $\left({ }^{14} \mathrm{C}\right)$ atrazine residue. Toxicol Environ Chem 26, 203-210

Boesten JJTI (1987) Leaching of herbicides to ground water: A review of important factors and of available measurements. 1987 British Crop Protection Conference-Weeds, 559-568 
Brambilla A, Rindone B, Polesello S, Galassi S, Balestrini R (1993) The fate of triazine pesticides in the River Po water. The Science of the Total Environment 132, 339-348

Calvet R (1989) Adsorption of organic chemicals in soils. Environmental Health Perspectives 83, 145177

Capriel P, Haisch A, Khan SU (1985) Distribution and nature of bound (nonextractable) residues of atrazine in a mineral soil nine years after the herbicide application. J Agric Food Chem 33, 567-569

Chambre d'agriculture de Lorraine (1992) Recherche de pesticides dans des captages d'adduction d'eau potable en Lorraine ; Campagne 1991. Rapport régional, $13 p$

Cohen SZ, Creeger SM, Carsel RF, Enfield CG (1984) Potential for pesticide contamination of groundwater from agricultural uses. Treatment and Disposal of Pesticides Wastes. ACS Symposium series 259, Am Chem Soc (RF Kruger, JN Sieber, eds), Washington, DC, 297-325

Copin A, Deleu R (1985) Les résidus de produits phytosanitaires dans le sol. Ann Gembloux 91, 89-101

Coste CM (1990) Quelques aspects quantitatifs et qualitatifs de la pollution des sols agricoles et des aquifères par les produits phytosanitaires. Communication AFES : Pollution des sols par les molécules organiques xénobiotiques, Paris, 22 mai 1990

Demon M (1994) Influence des facteurs climatiques et des constituants du sol sur la dynamique de l'atrazine. Thèse INPL-Nancy, $115 p$

Dictor MC (1994) Caractérisation de la distribution et du comportement métabolique de la microflore indigène dans un profil de sol. Thèse INPL-Nancy, $104 \mathrm{p}$

Donati A, Giolitti A, Marchettini N, Rossi C, Tiezzi E, Ulgiati S (1993) Environmental aspects of pesticide use in Italian agriculture. The Science of the Total Environment 129, 125-135

Gillet H (1992) Les triazines dans l'eau : état des lieux en Bretagne. ANPP-XVe Conférence Columa, Versailles 2-3-4 décembre 1992, 55-65

Golab T, Althaus WA, Wooten HL (1979) Fate of $\left[{ }^{14} \mathrm{C}\right]$ trifluralin in soil. J Agric Food Chem 27, 163-179

Golab T, Amundson ME (1975) Degradation of trifluralin, oryzalin, and isopropalin in soil. Environ Qual Safety suppl III, 258-261

Hall JK (1974) Erosional losses of s-triazine herbicides. J Environ Qual 3, 174-180

Hall JK, Hartwig NL, Hoffman LD (1983) Application mode and alternate cropping effects on atrazine losses from a hillside. J Environ Qual 12, 336-340

Hall JK, Pawlus M, Higgins ER (1972) Losses of atrazine in runoff water and soil sediment. $J$ Environ Qual 1, 172-176

Halleberg GR (1989) Pesticide pollution of groundwater in the humid United States. Agriculture, Ecosystems and Environment 26, 299-367
Helling CS, Krivonak EAE (1978) Physicochemical characteristics of bound dinitroaniline herbicides in soils. J Agr Food Chem 1156-1163

Huang LQ, Frink CR (1989) Distribution of atrazine, simazine, alachlor, and metolachlor in soil profiles in Connecticut. Bull Environ Contam Toxicol 43, 159164

Jarczyk HJ (1987) Studies on the leaching characteristics of crop protection chemicals in a monolith lysimeter installation. Pflanzenschutz-Nachrichten Bayer 40, 49-77

Jarczyk HJ (1983) Investigations on the leaching behaviour of herbicides under natural agronomic conditions of lysimeter-monoliths. Aspects of Applied Blology 4, 441-448

Kenimers AL, Mostaghimi S, Dillaha TA, Shanholtz VO (1989) PLIERS: Pesticide losses in erosion and runoff simulator. American Society of Agricultural Engineers 32, 127-136

Kloskowski R, Führ F, Mittelstaedt W (1987) The uptake of non-extractable soil-bound pesticide residues by roots-standardized experiments with 4 pesticides. Pesticide Science and Biotechnology (Greenhalgh, Roberts, eds), 405-410

Kreuger JK, Brink N (1988) Losses of pesticides from agriculture. Pesticides: food and environmental implications. IAEA-SM 297/21, 101-112

Kruger EL, Somasundaram L, Kanwar S, Coats JR (1993) Persistence and degradation of [ $\left.{ }^{14} \mathrm{C}\right]$ atrazine and $\left[{ }^{14} \mathrm{C}\right]$ deisopropyl-atrazine as affected by soil depth and moisture conditions. Environ Toxicol Chem 12, 1959-1967

Kubiak R, Führ F, Mittelstaedt W, Hansper M, Steffens $W$ (1988) Transferability of lysimeter results of actual field situations. Weed Sci 36, 514-518

Legrand MF, Costentin E, Bruchet A (1991) Occurrence of 38 pesticides in various French surface and ground waters. Environmental Toxicology 12, 985-996

Leonard RA, Langdale GW, Fleming WG (1979) Herbicide runoff from upland piedmond watersheds - data and implications for modeling pesticide transport. J Environ Qual 8, 223-229

Liu LC, Fernandez-Horta D, Santiago-Cordova M (1985) Diuron and ametryn runoff from a plantain field. J Agric Univ Puerto Rico 2, 177-183

Madhun YA, Young JL, Freed VH (1986) Binding of herbicides by water-soluble organic materials from soil. J Environ Qual 1, 64-68

Maguire RJ, Tkacz JR (1993) Occurrence of pesticides in the Yamaska River, Quebec. Arch Environ Contam Toxicol 25, 220-226

Montiel A (1990) Les micropolluants organiques rencontrés dans les eaux souterraines. Communication AFES : pollution des sols par les molécules organiques xénobiotiques, Paris, 22 mai 1990

Mudd PJ, Hance RJ, Wright SJL (1983) The persistence and metabolism of isoproturon in soil. Weed Research 23, 239-246

My J (1990) Qualité des eaux et pesticides. Phytoma 422,20 
Paterson KG, Schnoor JL (1992) Fate of alachlor and atrazine in a riparian zone field site. Water Environment Research 64, 274-283

Perrin-Ganier C, Schiavon M, Portal JM, Breuzin C (1994) Lessivage et distribution des résidus d'isoproturon au cours du temps. XXIV Congrès du groupe français des pesticides, Bordeaux 18-19 mai 1994, 19-22

Portal JM, Babut M, Schiavon M, Gérard B (1994) Échantillonnage et appréciation de la contamination de l'eau d'un point de captage par les produits phytosanitaires. Journal Européen d'Hydrologie 25, 135-152

Portal JM, Babut M, Schiavon M (1992) État de contamination des eaux du captage de Sainte-Geneviève (54) par différents produits phytosanitaires. Colloque PHYTEAU : EaU - Produits Phytosanitaires - Usages agricoles et connexes, Versailles 21-22 octobre 1992, 19-24

Réal B, Massé J, Gaillardon P, Arlot MP, Gril JJ, Gouy $V$ (1993) Reducing the impact on the environment of agricultural pesticides. Brighton Crop Protection. Weeds 867-872

Ritter WF, Johnson HP, Lovely WG, Molnau M (1974) Atrazine, propachlor, and diazinon residues on small agricultural watersheds. Environ Sci Technol $8,38-42$

Sauer TJ, Daniel TC (1987) Effect of tillage system on runoff losses of surface-applied pesticides. Soil Sci Soc Amer J 51, 410-415

Schiavon M (1988) Studies of the leaching of atrazine, of its chlorinated derivatives, and of hydroxyatrazine from soil using $\left[{ }^{14} \mathrm{C}\right]$ ring-labeled compounds under outdoor conditions. Ecotox Environ Safety 15, 46-54

Schiavon M (1980) Contribution à l'étude du mouvement et de la dégradation de l'atrazine dans 2 sols agricoles drainés. Interaction matière organiqueherbicide. Thèse d'État, Nancy, $193 p$
Schiavon M, Babut M, Portal JM (1992) Contamination des eaux de drainage des sols par les produits phytosanitaires. CR du XXI/e Congrès du GFP, Dijon, 19-20 mai 1992, 211-215

Schiavon M, Barriuso E, Portal JM et al (1990) Étude du devenir de 2 substances organiques utilisées dans les sols, l'une massivement (l'atrazine) et l'autre à l'état trace (le metsulfuron-méthyl), à l'aide de molécules marquées au $\left[{ }^{14} \mathrm{C}\right]$. Rapport SRETIE/MERE, $75 \mathrm{p}$

Schiavon M, Jacquin F (1973) Étude de la présence d'atrazine dans les eaux de drainage. CR des Journées d'étude sur les herbicides, COLUMA, Versailles 13-14 décembre 1973, 35-43

Southwick LM, Willis GH, Bengston RL, Lormand TJ (1990) Atrazine and metolachlor in subsurface drain water in Louisiana. J Irrigation Drainage Engineering 116, 16-23

Triplett GB, Conner BJ, Edwards WM (1978) Transport of atrazine and simazine in runoff from conventional and no-tillage corn. J Environ Qual 7, 77-84

Wauchope RD (1987) Tilted-bed simulation of erosion and chemical runoff from agricultural fields. II. Effects of formulation and atrazine runoff. J Environ Qual 16, 212-216

Wehtje GR, Spalding RF, Burnside OC, Lowry SR, Leavitt JR (1983) Biological significance and fate of atrazine under aquifer conditions. Weed Sci 31, $610-618$

Wheeler W, Stratton GD, Twilley RR, Li-Tse OU, Carlson DA (1979) Trifluralin degradation and binding in soil. J Agric Food Chem 27, 702-706

White AW, Barnett AP, Wright BG, Holladay JH (1967) Atrazine losses from fallow land caused by runoff and erosion. Environ Sci Technology 1, 740-744

Wu TL (1980) Dissipation of the herbicides atrazine and alachlor in a Maryland corn field. J Environ Qual 9, 459-465 